\title{
Hemorragia digestiva: evaluación por angio-TC y entero-TC
}

\section{Digestive bleeding: evaluation by CT angiography and CT enterography}

\author{
Andrés O’Brien ${ }^{1 *}$, Alex Wash-Franulic ${ }^{1}$, Omar Enríquez-Gutiérrez ${ }^{1}$, Sebastián Yevenes-Aravena ${ }^{1}$, \\ Gonzalo Zapata-Fagerstrom², M $^{a}$ Ignacia Labbe-Alessandrini², Francisca Garrido ${ }^{2}$ y Joel Fuentes ${ }^{2}$
}

${ }^{1}$ Servicio de Radiología; ${ }^{2}$ Servicio de Imágenes Abdominales. Clínica Las Condes, Santiago, Chile

\begin{abstract}
Resumen
El sangrado gastrointestinal es frecuente, resolviéndose en la mayoría de los casos de forma espontánea. Sin embargo, este puede ser intermitente y en algunos casos masivo, con compromiso hemodinámico del paciente y riesgo vital. En este sentido es muy importante realizar un diagnóstico rápido y certero del sitio y causa del sangrado, para poder tratarlo en forma adecuada. El diagnóstico es en ocasiones difícil dada la longitud del tubo digestivo y la intermitencia del sangrado, lo que lleva a la realización de múltiples exámenes, muchos de ellos invasivos y costosos. La endoscopia ha sido el estudio de primera línea para la evaluación del sangrado digestivo, en especial alto. Para el sangrado activo bajo, por su rapidez, disponibilidad y rendimiento, la angiografía por tomografía computarizada ha adquirido un rol promisorio y creciente, propuesto en muchos centros como test de primera línea, para posteriormente de acuerdo con su resultado proseguir con endoscopia dirigida, cirugía, angiografía u otros. La enterografía por tomografía computarizada se recomienda para sangrado digestivo estable $u$ oculto, con estudios endoscópicos negativos.
\end{abstract}

Palabras clave: Sangrado. Gastrointestinal. Angio-TC. Entero-TC.

\section{Abstract}

Gastrointestinal bleeding is frequent, resolving spontaneously in most cases. However, it can be intermittent or massive, leading to unstable hemodynamics, and risk of death. It is very important to get a fast and accurate diagnosis of site and cause of the bleeding, in order to treat it promptly. Due to the length and intermittency of the bleeding, sometimes multiple examinations are performed, leading to invasiveness and high costs to the patient. Endoscopy has been the standard of care for years, especially for high gastrointestinal bleeding. For active low Gl bleeding, we recommend CT angiography as the first line study, because it is fast and accurate, and depending on the results continue with targeted endoscopy, surgery, angiography or others. CT enterography is recommended for stable or occult bleeding with negative endoscopic studies.

Key words: Gl bleeding. CT angiography. CT enterography.

\section{Introducción}

El sangrado gastrointestinal es frecuente, siendo el agudo 3-6 veces más común en el tracto digestivo alto ${ }^{1}$. En la mayoría de los casos se resuelve en forma espontánea (80-85\%); sin embargo, en algunos casos puede ser intermitente o manifestarse con un sangrado masivo (más de $500 \mathrm{ml}$ en forma aguda), que puede llevar a inestabilidad hemodinámica y shock (cuando se ha perdido el $15 \%$ de la volemia) ${ }^{2}$. La evaluación
Correspondencia:

*Andrés O'Brien

E-mail: obrienandres@yahoo.com

0717-9308 / @ 2020 Sociedad Chilena de Radiología. Publicado por Permanyer. Éste es un artículo open access bajo la licencia CC BY-NC-ND (https://creativecommons.org/licenses/by-nc-nd/4.0/).
Disponible en internet: 21-09-2021 Rev Chil Radiol. 2021;27(3):132-138 www. resochradi.com 
clínica inicial es muy importante, para determinar el origen alto o bajo, la cuantía y tasa del sangrado, y los factores de riesgo del paciente, lo que ayudará en la determinación del mejor examen diagnóstico que realizar.

Según la nueva nomenclatura, se define sangrado alto cuando es proximal al ángulo de Treitz, bajo cuando es colorrectal y sangrado con probable origen desde intestino delgado cuando los estudios endoscópicos alto y bajo son negativos ${ }^{3}$. Sangrado digestivo oscuro se define como el sangrado cuyo origen no es encontrado pese a numerosos exámenes que incluyen la totalidad del tubo digestivo ${ }^{4}$. Sangrado manifiesto 0 evidente es el sangrado visible como hematemesis, melena o hematoquecia. Sangrado oculto es el sangrado no visible, que se infiere por anemia ferropriva o test de sangre oculta en deposiciones positivo.

El diagnóstico debe ser rápido y certero, logrando definir el sitio y la causa del sangrado, de manera que pueda tratarse adecuadamente. Esto requiere un enfoque multidisciplinario, con participación de gastroenterólogos, cirujanos, radiólogos diagnósticos e intervencionales, y médicos nucleares, entre otros.

Las causas del sangrado dependen de la edad y de si el sangrado es alto o bajo. Dentro de las causas de sangrado alto están, en orden de frecuencia: la úlcera péptica, lesiones vasculares, gastritis o duodenitis y várices. Las causas de sangrado bajo incluyen la enfermedad diverticular, enfermedad inflamatoria intestinal, neoplasias, coagulopatías, lesiones vasculares y congénitas como el divertículo de Meckel.

Dada la longitud del tubo digestivo y la intermitencia del sangrado en muchos casos, es difícil lograr un diagnóstico certero en forma rápida, llevando al paciente a una escalada de innumerables exámenes, algunos de ellos invasivos y costosos, como la enteroscopia con doble balón, la cápsula endoscópica y la angiografía fluoroscópica.

La endoscopia ha sido por años y es actualmente el método diagnóstico de elección para pacientes con hemorragia digestiva, mediante la cual puede diagnosticarse y tratarse la causa de la hemorragia en un mismo tiempo. Siempre está indicada en caso de hemorragia digestiva alta. En caso de hemorragia de origen bajo, si bien la endoscopia está también indicada, cuando el sangrado es activo con estabilidad o inestabilidad hemodinámica marginal es necesario realizar un diagnóstico rápido, por lo que otros estudios como la angiografía por tomografía computarizada (angio-TC) adquieren relevancia, dado que no necesita preparación y puede detectar la causa tanto en intestino delgado como en colon y recto. La enterografía por tomografía computarizada (entero-TC) está indicada en sangrado digestivo estable u oculto, con estudios endoscópicos alto y bajo negativos.

A continuación revisaremos las ventajas y desventajas, indicaciones, protocolos y diferentes casos que muestran la importancia de ambos exámenes, uno de ellos con resolución guiada bajo radiología intervencional.

\section{Angiografía por tomografía computarizada}

La angio-TC es una TC con múltiples detectores realizada antes y después de la administración de contraste endovenoso en distintas fases, lo que permite detectar en forma dinámica la presencia de un sangrado activo. Es un examen rápido, disponible, sistemático, de alto rendimiento en la detección del sitio y la causa del sangrado, con sensibilidad y especificidad superiores al $95 \% 5,6$, detectando tasas de sangrado superiores a 0,3-0,5 ml/min. Además, permite realizar reformateo multiplanar con una alta resolución espacial y no requiere preparación. Otra ventaja muy importante es que puede evaluar otros órganos abdominales en el mismo examen, permitiendo la detección de complicaciones, comorbilidades e incluso detectar causas de sangrado no originadas en el intestino, como la hemobilia. Entre las desventajas se incluyen: el uso de radiación ionizante, que con los avances tecnológicos es cada vez menor, en especial con los tomógrafos de doble energía; la utilización de contraste endovenoso, con los riesgos de nefropatía y reacciones alergoides; y la ausencia de distensión de asas intestinales, que disminuye la sensibilidad en la detección de tumores u otras causas.

Las indicaciones incluyen estudio de sangrado digestivo bajo activo, sangrado alto con estudio endoscópico frustro y estudio anatómico vascular arterial y venoso.

El protocolo incluye una fase sin contraste, una fase arterial realizada con una tasa de inyección de 4-5 cc/ segundo, que se gatilla cuando la densidad de la aorta abdominal superior alcanza un valor de $150 \mathrm{UH}$ (unidades Hounsfield), y una fase venosa $70-80$ segundos después de la administración de 100-125 ml de contraste, con una concentración de al menos $300 \mathrm{mg}$ de yodo/ml.

El sangrado activo se visualiza como un jet, una nube de contraste 0 un nivel líquido-contraste en fase arterial con una densidad superior a $90 \mathrm{UH}$, que aumenta de tamaño en la fase portal por la acumulación de contraste (Figs. 1 y 2) y no se visualiza en la 


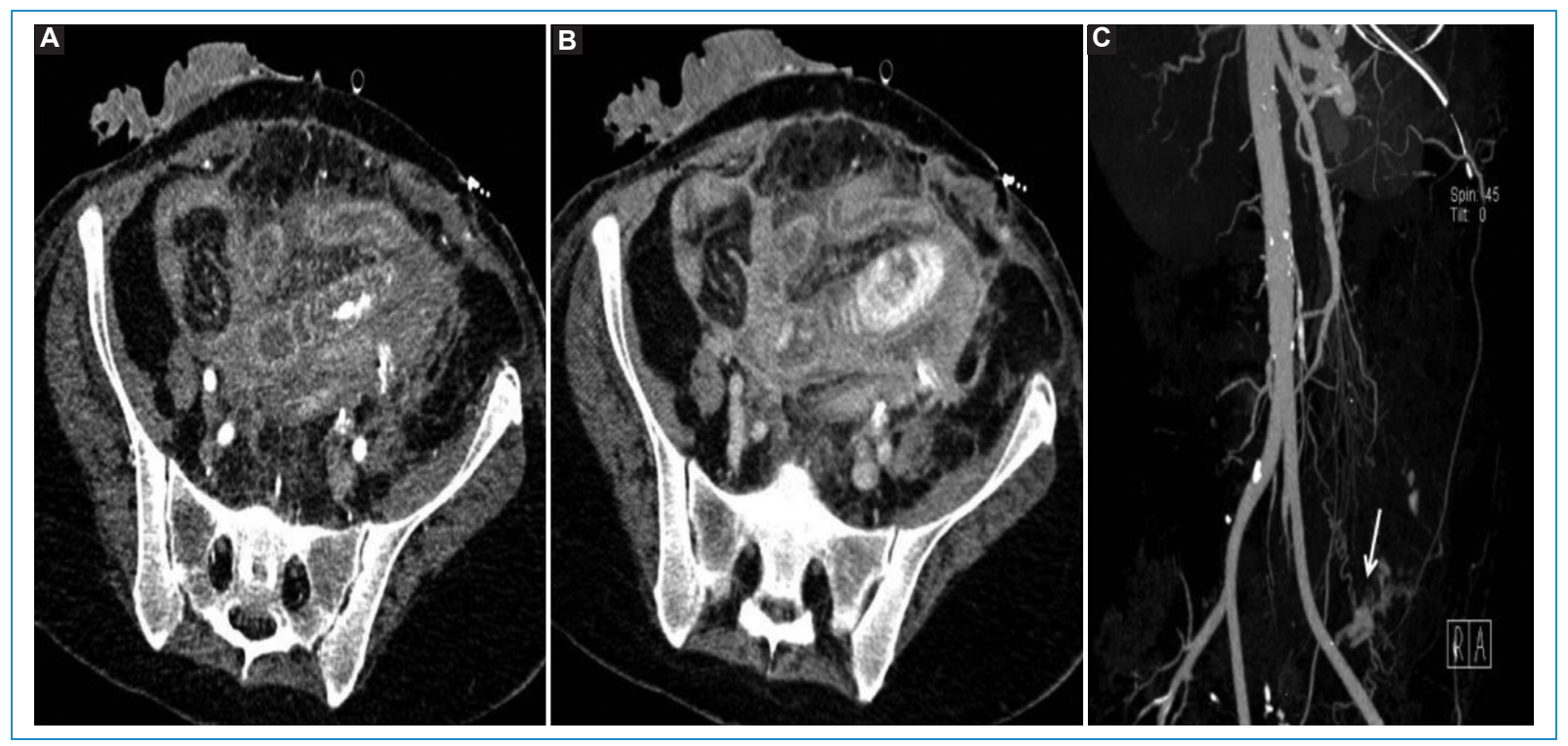

Figura 1. Sangrado activo de íleon secundario a isquemia. Mujer de 54 años con antecedente de diverticulitis aguda complicada, con hemicolectomía izquierda, ingresa por sangrado bajo masivo, con inestabilidad hemodinámica. A: angio-TC en fases arterial axial, B: venosa axial y C: reconstrucción MIP muestra sangrado activo en asa de íleon (flecha). Cirugía posterior demostró isquemia intestinal de 1 metro de longitud. Angio-TC: angiografía por tomografía computarizada; MIP: proyección de intensidad máxima.

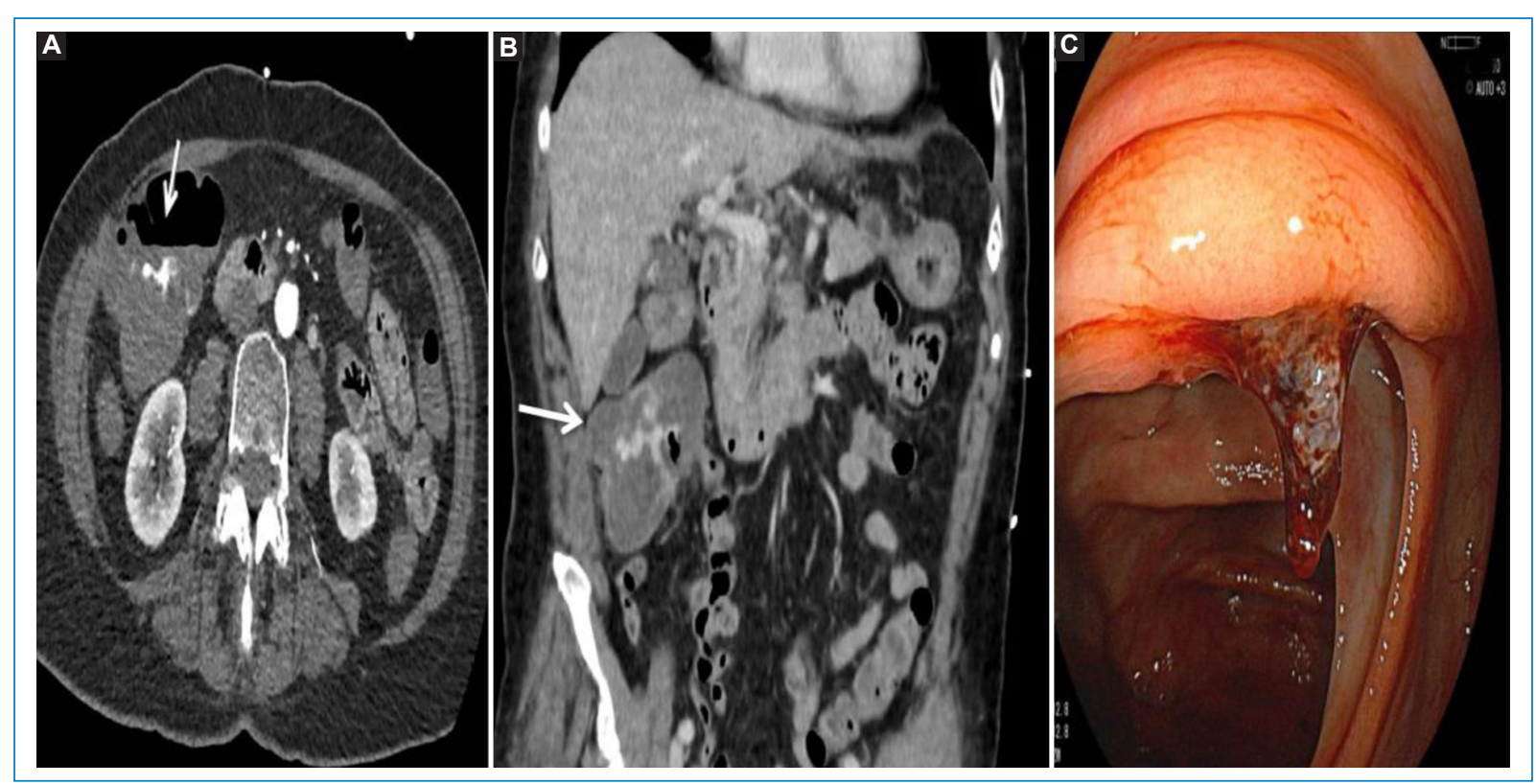

Figura 2. Sangrado activo de colon ascendente pospolipectomía reciente en mujer de 67 años. A: angio-TC en fases arterial axial y B: venosa coronal que muestra sangrado activo desde sitio de polipectomía en colon ascendente (flechas). C: se realiza endoscopia y se trata el sangrado.

Angio-TC: angiografía por tomografía computarizada.

fase sin contraste. Se sugiere realizar reconstrucciones 3D MIP (proyección de intensidad máxima), obteniendo un mapa del origen del sangrado, tanto en fase arterial como venosa, lo que aumenta la sensibilidad en la detección del foco del sangrado activo y sirve para planificar el tratamiento. Cuando la tasa de sangrado es baja, este puede visualizarse solo en la fase portal. $A$ veces, cuando el sangrado es intermitente, lo que se 
detecta es el coágulo centinela adyacente al sitio de sangrado en fase sin contraste (Fig. 3), pero sin apreciar signos de sangrado activo.

Como una de las desventajas de la angio-TC es el uso de radiación ionizante, se han utilizado distintas estrategias para su reducción. En primer lugar, se puede realizar la fase sin contraste con reducción de dosis; se puede obviar la fase arterial y solo hacer la portal, dado que tiene mayor sensibilidad ${ }^{7}$, o bien, si se utiliza un tomógrafo de doble energía, se puede obviar la fase sin contraste y obtener una fase virtual sin contraste al digitalmente eliminar el yodo ${ }^{8}$, e incluso obtener un mapa de yodo en que el sangrado se visualiza directamente.

\section{Enterografía por tomografía computarizada}

La entero-TC es una TC con múltiples detectores realizados en fases arterial y portal, luego de la ingestión de $1.500 \mathrm{cc}$ de contraste negativo (manitol al $3 \%$ ) previo al examen, logrando con esto una adecuada distensión de asas intestinales. Esto permite un adecuado contraste entre la pared, que es hiperdensa por el contraste endovenoso, y el lumen, que es hipodenso por el manitol. Tiene un alto rendimiento en la detección de patología de intestino delgado, con sensibilidad, especificidad y valor predictivo negativo superiores al $94 \%$, 10 .

Es un examen disponible, rápido, con alta resolución espacial, que permite no solo visualizar el lumen y la pared, sino también el mesenterio y órganos vecinos.

Entre las desventajas se cuentan el uso de radiación ionizante y contraste endovenoso, y una menor sensibilidad que la angio-TC en caso de sangrado activo por dilución del contraste extravasado con el manitol intraluminal.

Está indicada en estudio de enfermedad inflamatoria intestinal, enfermedad celíaca, neoplasias de intestino delgado, obstrucción intestinal parcial y hemorragia digestiva no activa u oculta con estudios endoscópicos alto y bajo negativos ${ }^{11}$.

El protocolo de examen consiste en administrar al paciente $1.500 \mathrm{cc}$ de manitol al $3 \%$ vía oral durante 1 hora, y posteriormente realizar una fase arterial tardía o enterográfica a los 35 segundos de inyectado el contraste endovenoso, y otra venosa a los 70 segundos.

Posteriormente los datos son analizados en una estación de trabajo, con reformateos coronales y reconstrucciones MIP.

Una de las principales causas de hemorragia digestiva en pacientes jóvenes es la enfermedad de Crohn, que puede manifestarse por dolor abdominal, masa palpable

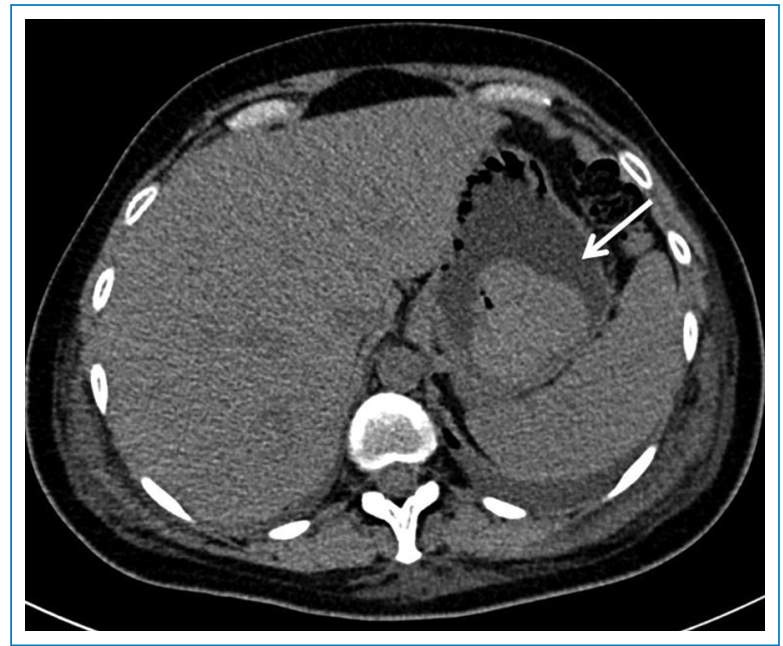

Figura 3. Coágulo centinela en fondo gástrico. Angio-TC en fase sin contraste axial muestra un coágulo en el fondo gástrico secundario a sangrado por lesión de Dieulafoy (flecha).

Angio-TC: angiografía por tomografía computarizada.

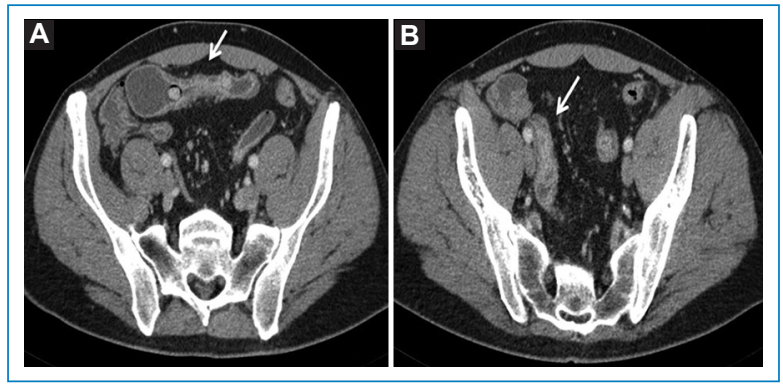

Figura 4. Enfermedad de Crohn con compromiso inflamatorio de íleon. A y B: cortes axiales de entero-TC en hombre de 38 años con dolor abdominal y anemia muestra engrosamiento parietal estratificado de íleon con proliferación del tejido adiposo mesentérico (flechas).

Entero-TC: enterografía por tomografía computarizada.

en fosa ilíaca derecha y/o sangrado digestivo crónico u oculto, con anemia o test positivo de sangre en deposiciones. El hallazgo principal es un engrosamiento parietal de íleon con estratificación de la pared e hiperrefuerzo mucoso, proliferación de la vasa recta y del tejido adiposo mesentérico adyacente, y adenopatías reactivas mesentéricas (Fig. 4). También pueden identificarse fístulas enteroentéricas, enterocólicas y enterocutáneas, y áreas de estenosis con dilatación proximal. Mediante la entero-TC puede realizarse seguimiento para evaluar la evolutividad; sin embargo, en pacientes jóvenes se 
prefiere la enterografía por resonancia magnética, dado que no utiliza radiación ionizante ${ }^{12}$ y pueden evaluarse mejor las fístulas perianales.

Las neoplasias intestinales, en especial cuando se ulceran, pueden causar sangrado digestivo agudo o crónico. Las principales son el adenocarcinoma, que se manifiesta como una lesión estenosante concéntrica en duodeno o yeyuno más frecuentemente; el tumor neuroendocrino 0 carcinoide, más común en íleon, que se manifiesta como uno o más nódulos o engrosamientos focales intestinales asociado a adenopatías espiculadas hipervasculares en mesenterio; el tumor estromal gastrointestinal, que se manifiesta como un nódulo submucoso que protruye al lumen y eventualmente al mesenterio, puede ulcerarse en un $50 \%$ determinando episodios de sangrado (Fig. 5) y no se asocia a adenopatías ${ }^{13}$; el linfoma, que puede ser primario o secundario, produce un engrosamiento parietal sin estratificación, que puede asociarse a «dilatación aneurismática» en vez de obstrucción, y a la presencia de adenopatías. La transición entre intestino normal y el engrosamiento es gradual (Fig. 6); y finalmente la metástasis, en general de primarios pulmonar, mamario o melanoma, que se asocia a invaginación.

Otra entidad que puede generar un sangrado intestinal es el síndrome de Peutz-Jeghers, que consiste en la presencia de múltiples pólipos hamartomatosos de intestino delgado y eventualmente gástricos y colónicos, asociados a manchas pequeñas de color oscuro en situación perioral, labios, boca y manos (Fig. 7). Estos pólipos pueden sangrar o producir anemia, y se asocian a un mayor riesgo de cánceres digestivos, ginecológicos, de pulmón y mama. Es secundario a una mutación del gen STK11.

Una causa muy frecuente de sangrado digestivo bajo es el secundario a lesiones vasculares, las más comunes son las angioectasias, y mucho menos frecuentes las lesiones arteriales como la lesión de Dieulafoy, las malformaciones arteriovenosas y las lesiones venosas como las várices. Las angioectasias se visualizan a la endoscopia como áreas puntiformes o parcheadas de eritema de 2 a $10 \mathrm{~mm}$ de tamaño, que corresponde a venas tortuosas delgadas que carecen de la capa elástica interna. En la entero-TC se visualizan como focos puntiformes 0 áreas discoides de refuerzo, menores a $5 \mathrm{~mm}$, mejor visualizadas en la fase entérica de contraste $^{14}$. También pueden presentar una apariencia bulbosa elongada en la pared intestinal, en especial del yeyuno (Fig. 8).

La lesión de Dieulafoy corresponde a una arteria que se localiza en la submucosa gástrica o intestinal, que protruye a través de un pequeño defecto mucoso, cuyo

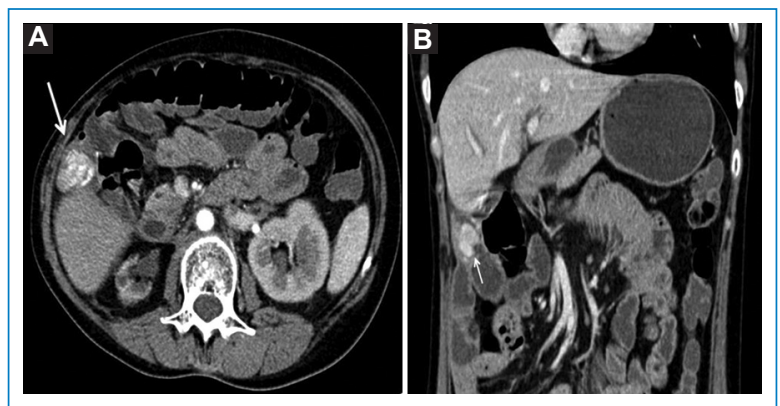

Figura 5. Tumor estromal gastrointestinal. A y B: cortes axiales de entero-TC de mujer de 45 años con anemia ferropriva, muestra nódulo submucoso ulcerado en íleon proximal (flechas).

Entero-TC: enterografía por tomografía computarizada.

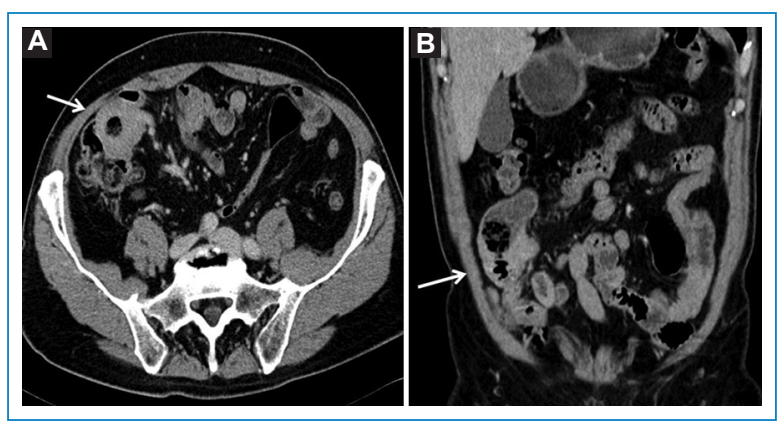

Figura 6. Linfoma MALT (tejido linfoide asociado a mucosas) de íleon. A: corte axial y B: reformateo coronal de entero-TC de hombre de 75 años con anemia ferropriva y distensión abdominal posprandial, muestra un engrosamiento masiforme focal de asa de íleon, no asociada a obstrucción (flechas).

Entero-TC: enterografía por tomografía computarizada.

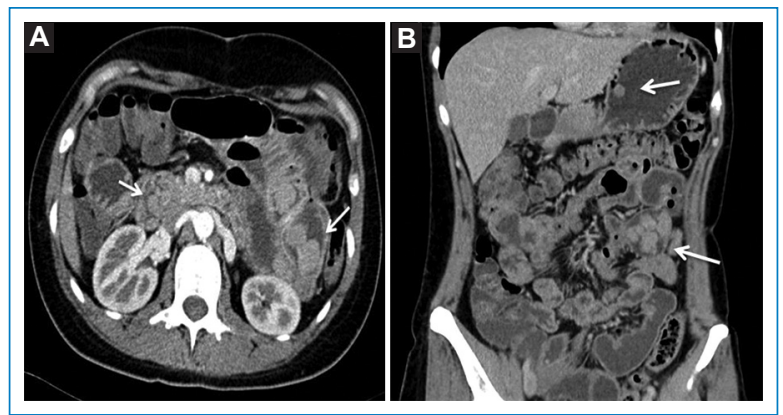

Figura 7. Síndrome de Peutz-Jeghers. A: corte axial y B: reformateo coronal de entero-TC de mujer de 30 años con pigmentación de piel y mucosa oral, y episodios de sangrado digestivo bajo, muestra la presencia de múltiples pólipos en intestino delgado, y uno gástrico (flechas).

Entero-TC: enterografía por tomografía computarizada. 


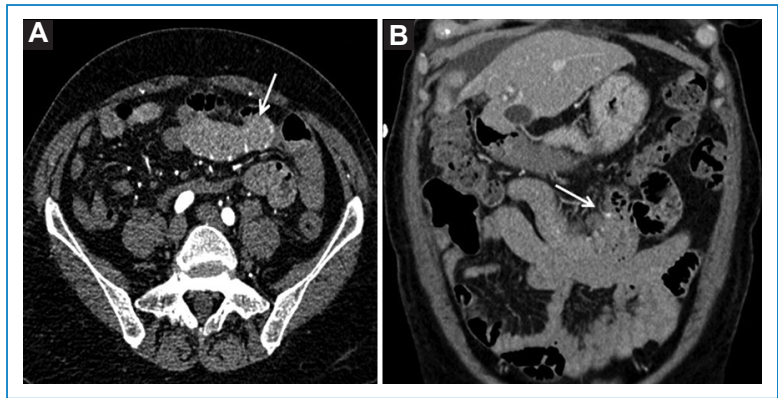

Figura 8. Angioectasias en yeyuno. A: corte axial en fase arterial y B: reformateo coronal en fase portal que demuestra la presencia de dilataciones venosas en la pared del yeyuno con las características de angioectasias (flechas).

MIP: proyección de intensidad máxima.

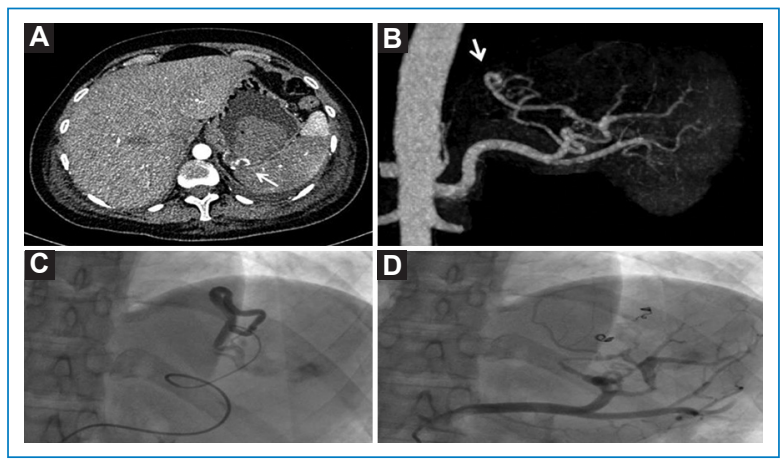

Figura 9. Lesión de Dieulafoy gástrica. A: corte axial de angio-TC en fase arterial y $\mathbf{B}$ : reconstrucción MIP muestra malformación vascular tipo lesión de Dieulafoy en el fondo gástrico asociado a coágulo centinela (flechas), C y D: que es tratado con embolización selectiva vía estudio angiográfico.

Angio-TC: angiografía por tomografía computarizada.

sangrado puede ser masivo, con riesgo vital. Son más frecuentes en el estómago (Fig. 9).

Por último, lesiones congénitas como el divertículo de Meckel pueden ser causa de sangrado digestivo bajo (Fig. 10), obstrucción intestinal o invaginación.

Una gran ventaja de la entero-TC es que visualiza todo el tubo digestivo (excepto el esófago), logrando identificar hallazgos incidentales que pueden explicar la sintomatología del paciente, como en el caso de la úlcera péptica (Fig. 11).

\section{Otros métodos diagnósticos}

La angio-TC y la entero-TC son complementarios y no reemplazan a otros estudios como la endoscopia,

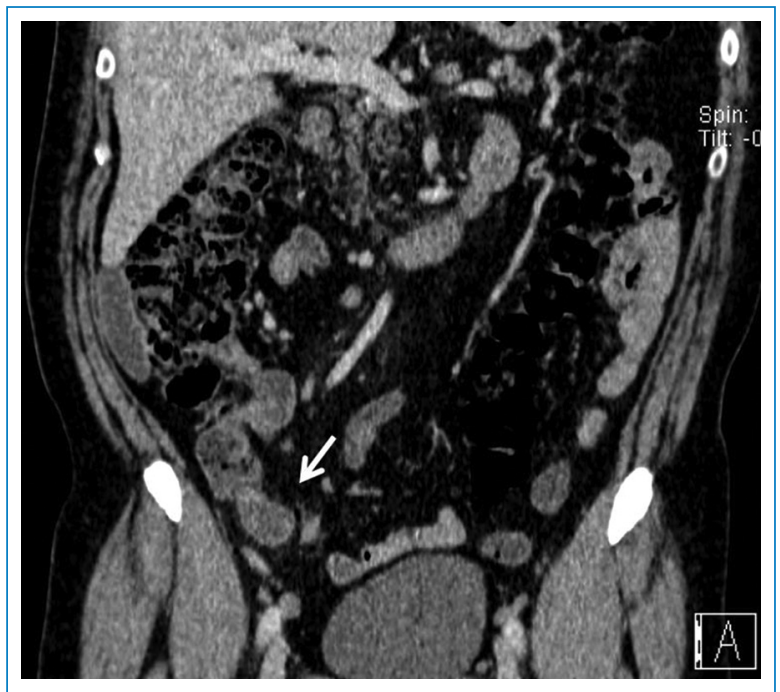

Figura 10. Divertículo de Meckel complicado. Reformateo coronal de entero-TC en hombre de 31 años muestra estructura tubular de fondo ciego de paredes engrosadas secundario a cambios inflamatorios (flecha). Entero-TC: enterografía por tomografía computarizada

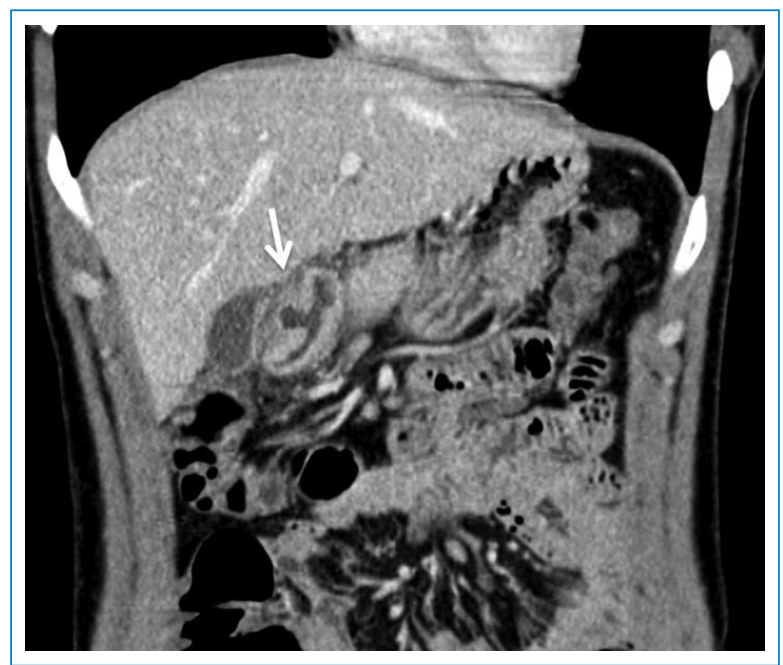

Figura 11. Úlcera en bulbo duodenal. Reformateo coronal de entero-TC de hombre de 25 años con dolor abdominal y anemia, muestra saliente de perfil compatible con úlcera en aspecto lateral del bulbo duodenal (flecha). Entero-TC: enterografía por tomografía computarizada.

cápsula endoscópica, enterografía de doble balón y cintigrafía con glóbulos rojos marcados.

La cápsula endoscópica tiene una alta sensibilidad en la detección de la causa del sangrado, visualizando la totalidad del tubo digestivo. Sin embargo, es costosa, el tiempo de examen es largo, su visión puede 
ser incompleta en un $20 \%$ y la cápsula puede quedar atascada por una estenosis, ya sea por enfermedad de Crohn, anastomótica o actínica.

La enterografía de doble balón es un examen que puede visualizar directamente el intestino delgado, y puede tratar la causa del sangrado; sin embargo, es muy costosa, poco disponible y en ocasiones es necesario utilizar las vías anterógrada y retrógrada.

La cintigrafía con glóbulos rojos marcados es la técnica más sensible para la detección del sangrado digestivo, logrando captar sangrados lentos e intermitentes de hasta 0,1-0,5 ml/min. Sus desventajas son su baja disponibilidad en horario inhábil y la mala definición anatómica dada su baja resolución espacial.

Por otro lado, la angiografía puede ser diagnóstica y terapéutica, detecta sangrados de 0,5-1 $\mathrm{ml} / \mathrm{min}$; sin embargo, es costosa, invasiva y puede generar isquemia.

Con los avances tecnológicos, y la necesidad de un diagnóstico rápido y certero, la angio-TC presenta un rol cada vez más central en el algoritmo de trabajo de la hemorragia digestiva baja activa de pacientes con estabilidad hemodinámica, o marginalmente inestables. Por esto se propone como estudio de primera línea en estos pacientes, y de acuerdo con su resultado se puede continuar con endoscopia, cirugía o angiografía en caso de ser positivo; o con otros estudios como la cápsula endoscópica o cintigrafía en caso de ser negativo. En los pacientes inestables hemodinámicamente se sugiere realizar de entrada angiografía o cirugía, y colonoscopia en los estables sin sangrado activo.

En conclusión, la hemorragia digestiva es un problema común, y la evaluación clínica es crucial para la elección del examen diagnóstico. Actualmente el método diagnóstico de elección es la endoscopia; sin embargo, el rol de la angio-TC es cada vez mayor y lo proponemos como estudio de primera línea en sangrado activo bajo con hemodinamia estable o marginalmente inestable. La entero-TC se recomienda en sangrado digestivo estable u oculto, con estudios endoscópicos alto y bajo negativos.

\section{Financiamiento}

Los autores declaran no haber recibido financiamiento para este estudio.

\section{Conflicto de intereses}

Los autores declaran no tener conflicto de intereses.

\section{Responsabilidades éticas}

Protección de personas y animales. Los autores declaran que para esta investigación no se han realizado experimentos en seres humanos ni en animales.

Confidencialidad de los datos. Los autores declaran que han seguido los protocolos de su centro de trabajo sobre la publicación de datos de pacientes.

Derecho a la privacidad y consentimiento informado. Los autores han obtenido el consentimiento informado de los pacientes $\mathrm{y} / \mathrm{o}$ sujetos referidos en el artículo. Este documento obra en poder del autor de correspondencia.

\section{Bibliografía}

1. Wells ML, Hansel SL, Bruining DH, Fletcher JG, Froemming AT, Barlow $\mathrm{JM}$, et al. CT for evaluation of acute gastrointestinal bleeding. Radiographics. 2018;38(4):1089-107.

2. Laing CJ, Tobias T, Rosenblum DI, Banker WL, Tseng L, Tamarkin SW. Acute gastrointestinal bleeding: emerging role of multidetector CT angiography and review of current imaging techniques. Radiographics. 2007;27(4):1055-70.

3. Strate LL, Gralnek IM. ACG clinical guideline: management of patients with acute lower gastrointestinal bleeding. Am J Gastroenterol. 2016;111(4):459-74.

4. Gerson LB, Fidler JL, Cave DR, Leighton JA. ACG clinical guideline: diagnosis and management of small bowel bleeding. Am J Gastroenterol. 2015;110(9):1265-87.

5. Wortman JR, Landman W, Fulwadhva UP, Viscomi SG, Sodickson AD. CT angiography for acute gastrointestinal bleeding: what the radiologist needs to know. Br J Radiol. 2017;90(1075):20170076.

6. Dobritz M, Engels HP, Schneider A, Bauer J, Rummeny EJ. Detection of intestinal bleeding with multi-detector row CT in an experimental setup. How many acquisitions are necessary? Eur Radiol. 2009;19:2862-9.

7. Dobritz M, Engels HP, Schneider A, Wieder H, Feussner H, Rummeny EJ, et al. Evaluation of dual-phase multi-detector-row CT for detection of intestinal bleeding using an experimental bowel model. Eur Radiol. 2009;19:875-81.

8. Sun H, Xue HD, Wang YN, Qian JM, Yu JC, Zhu F, et al. Dual-source dual-energy computed tomography angiography for active gastrointestinal bleeding: a preliminary study. Clin Radiol. 2013;68:139-47.

9. Boudiaf M, Jaff A, Soyer P, Bouhnik Y, Hamzi L, Rymer R. Small bowel diseases: prospective evaluation of multi-detector row helical CT enteroclysis in 107 consecutive patients. Radiology. 2004;233:338-44.

10. O'Brien A, Cruz JP, Berríos C, Melipillán Y, Butte JM, Álvarez M. Avances en radiología del intestino delgado: enteroclisis por tomografía computarizada. Gastroenterol Hepatol. 2006;29(9):528-33.

11. O'Brien A. Enteroclisis por tomografía computada. Rev Chil Radiol. 2006;12(2):70-5.

12. Estay C, Siamián D, Lubascher J, Figueroa C, O'Brien A, Quera R. Ionizing radiation exposure in patients with inflammatory bowel disease: are we overexposing our patients? J Dig Dis. 2015;16(2):83-9.

13. O'Brien A. Evaluación imaginológica del intestino delgado por TC y RM. Rev Med Clin Condes. 2013;24(1):109-15.

14. Huprich JE, Barlow JM, Hansel SL, Alexander JA, Fidler JL. Multiphase CT enterography evaluation of small-bowel vascular lesions. AJR Am J Roentgenol. 2013;201:65-72. 